\title{
Acetabular Version Increases After Closure of the Triradiate Cartilage Complex
}

\author{
Christoph E. Albers MD, Andrea Schwarz MD, Markus S. Hanke MD, \\ Karl-Philipp Kienle MD, Stefan Werlen MD, Klaus A. Siebenrock MD
}

Published online: 1 September 2016

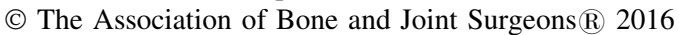

\begin{abstract}
Background Although the etiology of primary femoroacetabular impingement (FAI) is considered developmental, the underlying pathogenic mechanisms remain poorly understood. In particular, research identifying etiologic factors associated with pincer FAI is limited.
\end{abstract}

Each author certifies that he or she, or a member of his or her immediate family, has no funding or commercial associations (eg, consultancies, stock ownership, equity interest, patent/licensing arrangements, etc) that might pose a conflict of interest in connection with the submitted article.

All ICMJE Conflict of Interest Forms for authors and Clinical

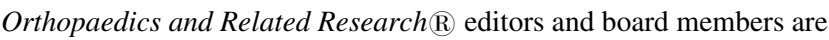
on file with the publication and can be viewed on request.

Clinical Orthopaedics and Related Research $\AA$ neither advocates nor endorses the use of any treatment, drug, or device. Readers are encouraged to always seek additional information, including FDAapproval status, of any drug or device prior to clinical use.

Each author certifies that his or her institution approved the human protocol for this investigation, that all investigations were conducted in conformity with ethical principles of research, and that informed consent for participation in the study was obtained.

Electronic supplementary material The online version of this article (doi:10.1007/s11999-016-5048-0) contains supplementary material, which is available to authorized users.

C. E. Albers ( $₫)$, A. Schwarz, M. S. Hanke, K.-P. Kienle, K. A. Siebenrock

Department of Orthopaedic Surgery, Inselspital, University Hospital Bern, Freiburgstrasse, 3010 Bern, Switzerland

e-mail: christoph.albers@insel.ch

C. E. Albers

Department of Orthopaedic Surgery, Fiona Stanley Hospital, Perth, Australia

S. Werlen

Department of Radiology, Sonnenhof Clinic, Bern, Switzerland
Knowledge of the physiologic growth patterns of the acetabulum during skeletal maturation might allow conclusions on deviations from normal development that could contribute to pincer-related pathomorphologies.

Questions/purposes In a population of healthy children, we asked if there were any differences related to skeletal maturation with regard to (1) acetabular version; (2) acetabular depth/width ratio; and (3) femoral head coverage in the same children as assessed by MRIs obtained 1 year apart.

Methods We prospectively compared 129 MRIs in 65 asymptomatic volunteers without a known hip disorder from a mixed primary/high school population (mean age, 12.7 years; range, 7-16 years). All participants underwent two MRI examinations separated by a minimum interval of 1 year. Based on the status of the triradiate cartilage complex (open versus closed [TCC]), all hips were allocated to the following groups: "open-open" = open TCC at both MRIs ( $\mathrm{n}=45$ hips [22 bilateral]); "openclosed" = open TCC at initial and closed TCC at followup MRI ( $\mathrm{n}=26$ hips [13 bilateral]); and "closed-closed" group $=$ closed TCC at both MRIs $(\mathrm{n}=58$ hips [29 bilateral]). We assessed acetabular version in the axial plane at five different locations $(5,10,15,20 \mathrm{~mm}$ below the acetabular dome and at the level of the femoral head) as well as three-dimensional (3-D) acetabular depth/width ratio and 3-D femoral head coverage on six radial MRI sequences oriented circumferentially around the femoral neck axis. Using analysis of variance for multigroup comparisons with Bonferroni adjustment for pairwise comparisons, we compared the results between the initial and followup MRI examinations and among the three groups.

Results Acetabular version was increased in hips of the "open-closed" group at the followup MRI compared with 
the initial MRI at $5 \mathrm{~mm}(-6 \pm 4.6[95 \%$ confidence interval $\{\mathrm{CI}\},-7.6$ to -3.6$]$ versus $-1 \pm 5.0[95 \% \mathrm{CI}$, -3.3 to 0.7 ]; $\mathrm{p}<0.001), 10 \mathrm{~mm}(0 \pm 4.0$ [95\% CI, -1.6 to 2.1$]$ versus $7 \pm 4.6$ [95\% CI, 4.4-8.7]; $\mathrm{p}<0.001$ ), and $15 \mathrm{~mm}(8 \pm 5.0[95 \% \mathrm{CI}, 6.1-10.2]$ versus $15 \pm 4.6[95 \%$ CI, 13.3-17.4]; $\mathrm{p}<0.001$ ) below the acetabular dome. Acetabular version did not change between the initial and followup MRI in the "open-open" and "closed-closed" groups. Independently of the groups, acetabular version was increased in all hips with a fused TCC compared with hips with an open TCC (mean difference measured at $5 \mathrm{~mm}$ below the acetabular dome at initial MRI examination: $2^{\circ} \pm 5.9^{\circ}\left[95 \% \mathrm{CI}, 0.2^{\circ}-3.4^{\circ}\right]$ versus $-9^{\circ} \pm 4.4^{\circ}$ [95\% CI, $-9.9^{\circ}$ to $\left.-7.8^{\circ}\right] ; \mathrm{p}<0.001$; at followup MRI examination: $1^{\circ} \pm 5.7^{\circ}\left[95 \% \mathrm{CI}, 0.1^{\circ}-2.7^{\circ}\right]$ versus $-9^{\circ} \pm$ $3.8^{\circ}\left[95 \% \mathrm{CI},-10^{\circ}\right.$ to $\left.\left.-7.6^{\circ}\right] ; \mathrm{p}<0.001\right)$. Both acetabular depth/width ratio and femoral head coverage did not differ among the groups or between the initial and followup MRI examinations within each group.

Conclusions Although acetabular depth/width ratio and femoral head coverage remain relatively constant, acetabular version increases with advancing skeletal maturity. There seems to be a relatively narrow timeframe near physeal closure of the TCC within which acetabular orientation changes to more pronounced anteversion. Further studies with greater numbers and longer followup periods are required to support these findings and determine whether such version changes may contribute to pincertype pathomorphologies.

Level of Evidence Level II, prospective study.

\section{Introduction}

Although understanding of the underlying pathologic mechanisms and treatment options for femoroacetabular impingement (FAI) continues to evolve [6], its etiology remains poorly understood. Several studies have reported an association between high-level athletic activity in adolescence and an increased prevalence of cam-type FAI, which they attribute to repetitive trauma to the proximal femoral physis during a critical period of time near physeal closure $[7,10,11,16,22,23]$. However, similar research identifying etiologic factors for pincer lesions is limited. Both acetabular retroversion and increased acetabular depth are potential causes for pincer-type impingement $[6,20]$.

A precise understanding of normal developmental changes of orientation and dimension of the acetabulum in the maturing skeleton may add to a better understanding of physiologic growth patterns, identification of deviations of normal development that subsequently lead to pathomorphologies associated with pincer-type FAI, and the application of rational treatment strategies that can take advantage of this knowledge. Acetabular development results from the confluence of the ilium, ischium, and pubic bones forming the $\mathrm{Y}$-shaped triradiate cartilage complex (TCC). A combination of interstitial, enchondral, and appositional bone formation determines the growth and dimensions (height, depth, width) of the acetabulum $[2,17,26]$. At approximately the age of 8 years, three secondary ossifications centers appear at the acetabular rim representing the epiphyses of the ilium, ischium, and pubic bones $[17,26]$.

Whereas most previous studies assessed developmental changes of the acetabulum in the context of hip dysplasia $[5,13,19,29]$, only two studies have evaluated acetabular version and coverage during skeletal development with regard to pincer-type FAI $[9,15]$, both of which noted that version and coverage were increased with increasing skeletal maturity $[9,15]$. However, both studies were based on a retrospective cross-sectional study design and data were derived from CT. Although CT offers the potential of excellent soft tissue to bone contrast properties, cartilaginous portions of the acetabular rim in skeletally immature patients may be underrepresented. Because MRI is superior in depicting these nonossified portions of the acetabular rim in skeletally immature subjects $[4,21]$, it is a good tool for analyzing acetabular growth and development.

In this prospective, observational study of asymptomatic volunteers in varying stages of skeletal maturity, we therefore asked the following questions: Are there any differences related to skeletal maturation with regard to (1) acetabular version; (2) acetabular depth/width ratio; and (3) femoral head coverage in the same children as assessed by MRIs obtained 1 year apart?

\section{Patients and Methods}

Three hundred thirty-one healthy students (age range, 618 years) from a mixed primary and high school population were contacted and invited to participate in this longitudinal study to undergo two MRIs of both hips with a minimum interval of 1 year (mean, 1.02 year; range, 11.1 years) between both examinations. This same study population was previously assessed for morphologic features of the proximal femur as part of a different longitudinal study [12]. Ninety-two students (28\%) volunteered to participate. Of those, five students were excluded based on the exclusion criteria (hip pain: $\mathrm{n}=1$; history of previous hip disorder [slipped capital femoral epiphysis: $\mathrm{n}=1$; Legg-Calvé-Perthes disease: $\mathrm{n}=3$ ]). The resulting 87 students underwent baseline MRIs of both hips (mean age, 12.5 years; range, $6-16$ years; $51 \%$ male). 
At the 1-year followup mark, 15 participants refused the followup examination and four participants were lost to followup. One additional participant had sustained a fracture of the femur requiring operative fixation and was subsequently excluded from the study. This left a total of 67 participants $(77 \%$ of the original 87$)$ who underwent bilateral hip MRI at the 1-year followup mark. Of those, one participant refused the MRI of the contralateral hip as a result of claustrophobia and four MRIs (in two subjects) could not be used for further analyses as a result of motion artifacts. Ultimately, 129 complete baseline and 1-year followup MRIs were available for analysis (65 subjects; 33 male [32 bilateral; mean age, 12.8 years; range, 716 years]; 32 female [ 32 bilateral; mean age, 12.6 years; range, $7-15$ years]; Fig. 1). The local institutional review board approved this study.

MRI without contrast was performed using a 3-Tesla scanner (Siemens Medical Solutions, Erlangen, Germany). Subjects were positioned supine, feet first, with the lower extremities in holding devices to avoid motion artifacts and to ensure standardized posture. The MRI protocol has previously been described in detail [12]. In summary, the protocol included the acquisition of sagittal three-dimensional (3-D) T2-weighted SSFP sequences (TrueFISP) and radial turbo spin echo sequences arranged circumferentially around the femoral neck axis in a clockwise fashion with $30^{\circ}$ increments (Fig. 2). Identical MRI protocols were used at both the baseline and 1-year followup examinations.

All measurements were performed using the commercially available JiveX software (Version 4.7; VISUS Technology Transfer GmbH, Bochum, Germany). To assess skeletal maturity, the status of the TCC was assessed on cartilage-sensitive 3-D TrueFISP sagittal sequences (Fig. 3). TCC was considered "open" if at least one flange of the Y-shaped physis (represented by a hyperintense, linear signal through the acetabulum) was present corresponding to Level 1 (open) or Level 2 (partially ossified) according to the modified Oxford system ([24] Fig. 3). It was considered "closed" if the acetabulum appeared homogenously black without any intraosseous cartilage corresponding to Level 3 of the modified Oxford system ([24] Fig. 3). We defined three groups based on the status of the TCC: first, group "openopen": TCC open at initial and followup examination; second, group "open-closed": TCC open at initial examination and closed at followup examination; and third, group "closed-closed": TCC closed at initial and followup examination. Allocation to the groups was based on the status of the TCC at each MRI examination (Table 1). Each participant remained in the group during the complete phase of the study period. Also, in all but one study participant, bilateral MRI of the hip was performed, and there was no case with unilaterally closed

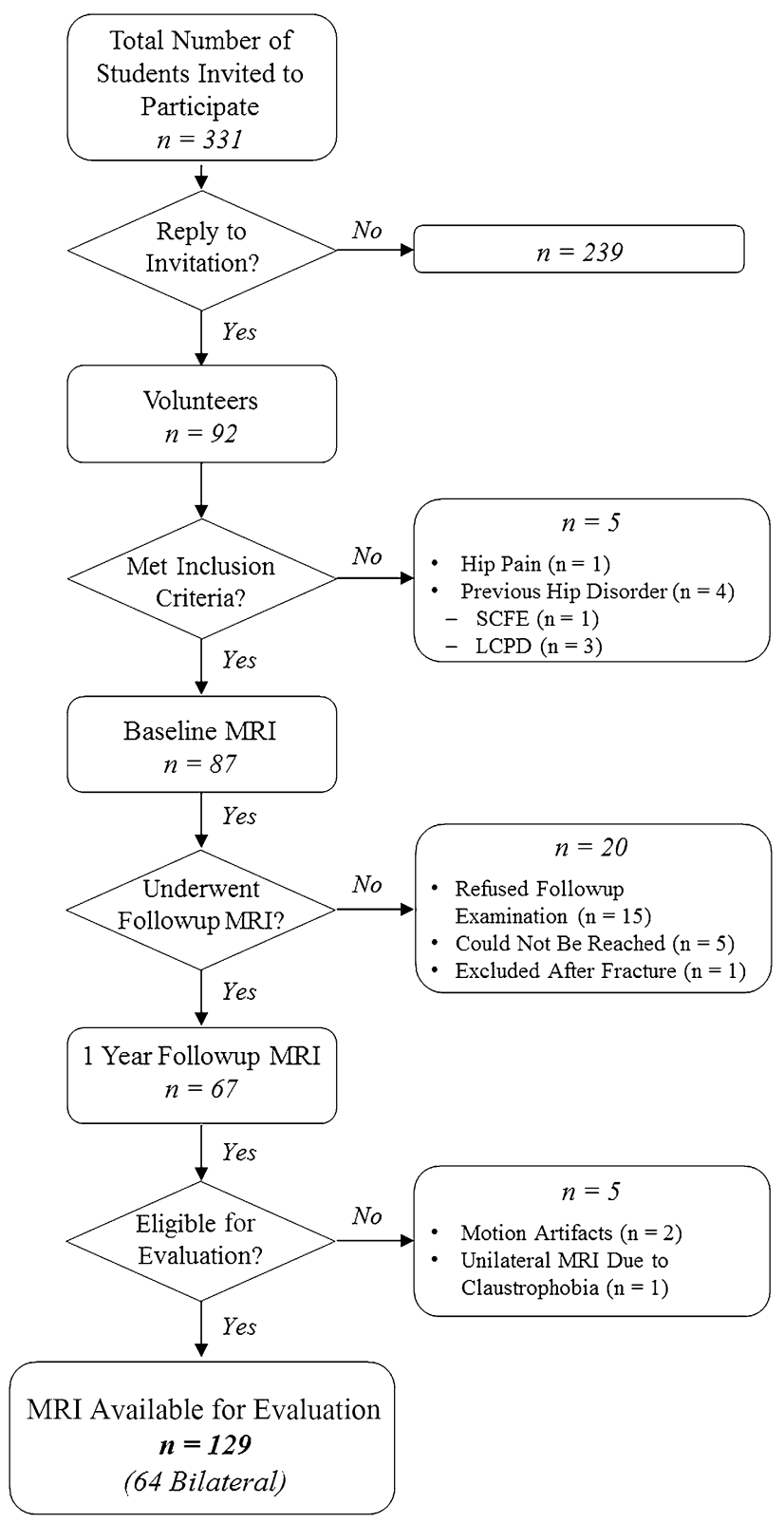

Fig. 1 Flowchart showing the recruitment and selection process of the study participants [12]. SCFE = slipped capital femoral epiphysis; LCPD = Legg-Calvé-Perthes disease.

TCC at the baseline or followup MRI examination affirming the integrity of the assigned study groups throughout the study period. The outcome parameters were assessed for intraindividual differences between the baseline and followup MRI examinations. Additionally, we evaluated interindividual differences among the three groups.

We measured acetabular version in the axial plane on reformatted transverse images (TrueFISP sequences) at four points through the acetabulum $(5 \mathrm{~mm}, 10 \mathrm{~mm}$, $15 \mathrm{~mm}$, and $20 \mathrm{~mm}$ below the acetabular dome) according 


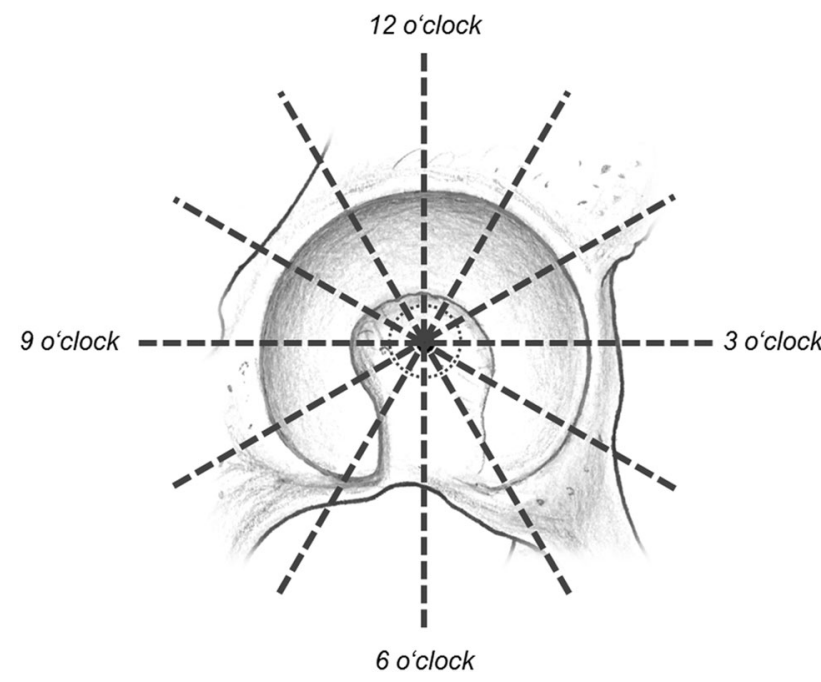

Fig. 2 A schematic drawing outlining MRI slices reconstructed radially around the femoral neck axis is shown. These reconstructions were used to three-dimensionally assess acetabular depth, acetabular width, and femoral head coverage. to the method by Hingsammer et al. [9]. An additional measurement was obtained at the level of the center of the femoral head [9]. The version angle was comprised of a line connecting the margins of the anterior and posterior acetabular rim and a sagittal reference line. To account for cartilaginous secondary ossification centers of the anterior and posterior acetabular rim in skeletally immature patients, the reference points of the line connecting the margins of the anterior and posterior acetabular rim were defined at the base of the labrum where it attaches to the acetabular rim ([4, 17] Fig. 4). Because the labrum is made of fibrocartilage, it has a distinctly different signal intensity on MRI compared with the epiphyseal articular cartilage at the secondary ossification centers of the acetabular rim, making it suitable for accurately measuring the margins of the acetabular wall in both skeletally mature and immature subjects (Fig. 4).

Acetabular depth was measured three-dimensionally according to the modified method by Rális and McKibbin
Fig. 3A-B Sagittal TrueFISP sequences through the TCC are shown (dotted circle $=$ bottom of fossa acetabuli). The TCC was considered "open" if at least one flange of the Y-shaped physis (represented by a hyperintense, linear signal through the acetabulum) was present (A). It was considered "closed" if the acetabulum appeared homogenously black without any corresponding intraosseous cartilage $(\mathbf{B})$.
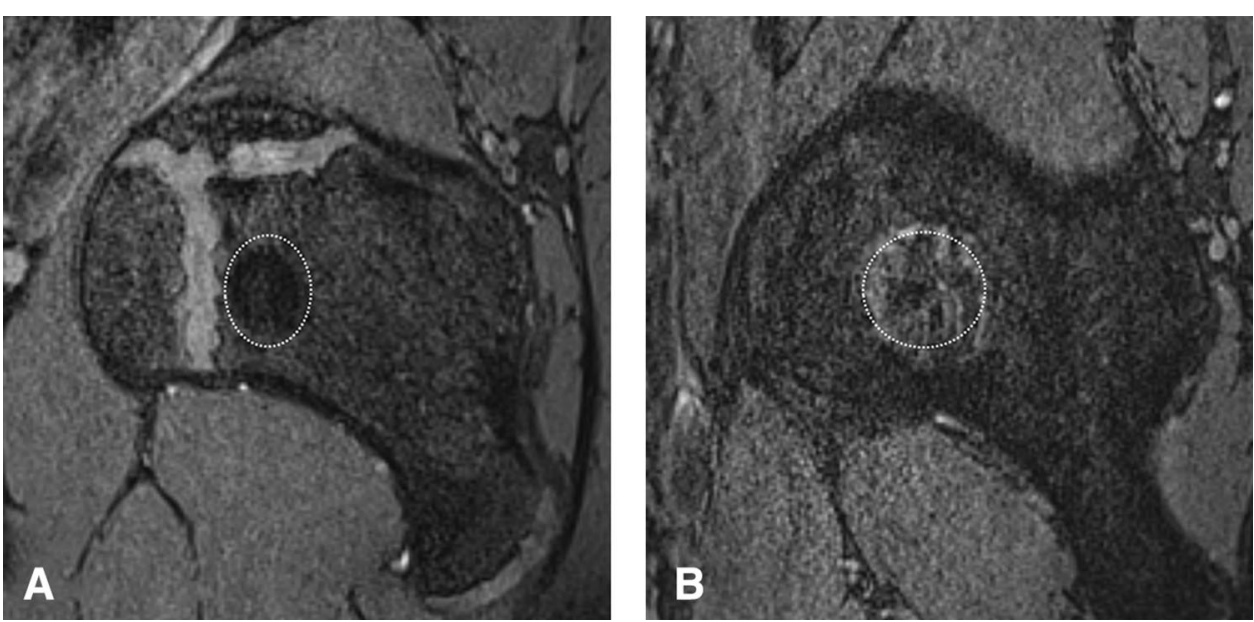

Table 1. Demographic distribution depending on the status of the TCC

\begin{tabular}{lllll}
\hline Parameter & TCC status & All & Female & Male \\
\hline Number of hips $(\%)$ & Open-open & $45(34)$ & $12(9)$ & $33(26)$ \\
Number bilateral & & 22 & 6 & 16 \\
Age (years) & & $10.9 \pm 1.8(7-13)$ & $9.7 \pm 2.2(7-12)$ & $11.3 \pm 1.5(7-13)$ \\
Number of hips $(\%)$ & Open-closed & $26(20)$ & $14(11)$ & $12(9)$ \\
Number bilateral & & 13 & 7 & 6 \\
Age (years) & & $12.7 \pm 0.9(11-14)$ & $38(29)$ & $13.2 \pm 0.7(12-14)$ \\
Number of hips $(\%)$ & Closed-closed & $58(45)$ & 19 & $20(16)$ \\
Number bilateral & & 29 & $13.7 \pm 1.0(11-15)$ & 10 \\
Age (years) & & $14.1 \pm 1.1(11-16)$ & $15.0 \pm 0.8(14-16)$ \\
\hline
\end{tabular}

TCC = triradiate cartilage complex; "open-open" = open TCC at both examinations; "open-closed" = open TCC at initial examination and closed TCC at followup examination; "closed-closed = closed TCC at both examinations; the indicated age refers to the age at date of the initial MRI examination; continuous values are expressed as mean \pm SD with range in parentheses. 


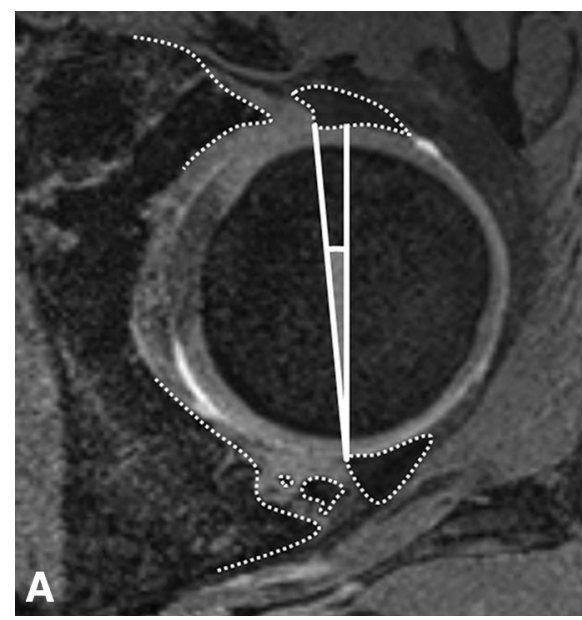

Fig. 4A-B Acetabular version was assessed on reformatted TrueFISP axial sequences. The measurements were conducted $5 \mathrm{~mm}$, $10 \mathrm{~mm}, 15 \mathrm{~mm}$, and $20 \mathrm{~mm}$ below the acetabular dome as well as at the center of the femoral head [9]. The acetabular version angle was formed by a line connecting the margins of the anterior and posterior

[19] on six axial slices (proton density-weighted sequences) arranged circumferentially around the femoral neck axis in $30^{\circ}$ increments (Fig. 2). Acetabular depth was defined as the distance between a line connecting both margins of the acetabular rim and a second line parallel to the first line and tangential to the deepest point of the acetabular socket (Fig. 5). The reference points for the first line were located at the base of the triangularly shaped labrum where it attaches to the acetabular rim to account for the entire extent of the acetabular rim. The length of the first line was defined as acetabular width. Acetabular shape was expressed as the ratio between the acetabular depth and width (Fig. 5). A depth/width ratio of 0.5 corresponds to a complete hemisphere.

Femoral head coverage was determined three-dimensionally also using the modified method of Rális and McKibbin [21] on six slices oriented circumferentially around the femoral neck axis in $30^{\circ}$ increments using proton density-weighted sequences (Fig. 2). Femoral head coverage was measured as the distance between a line connecting both margins of the acetabular rim and a second line parallel to the first line and located tangentially on the femoral head contour. The reference points of the line connecting both acetabular rims were defined at the base of the triangularly shaped labrum. Femoral head coverage was defined as the ratio of this distance and the femoral head diameter (Fig. 6).

Intra- and interobserver reproducibility and reliability were tested for the status of TCC (open TCC versus closed TCC) on 30 randomly chosen and blinded MRIs by two independent observers (CEA, MSH) on two separate occasions. High concordance was found for the status of the TCC (intraobserver 1 : $\kappa 0.93$; intraobserver $2: \kappa 1.0$; interobserver: $\kappa 0.97)$.

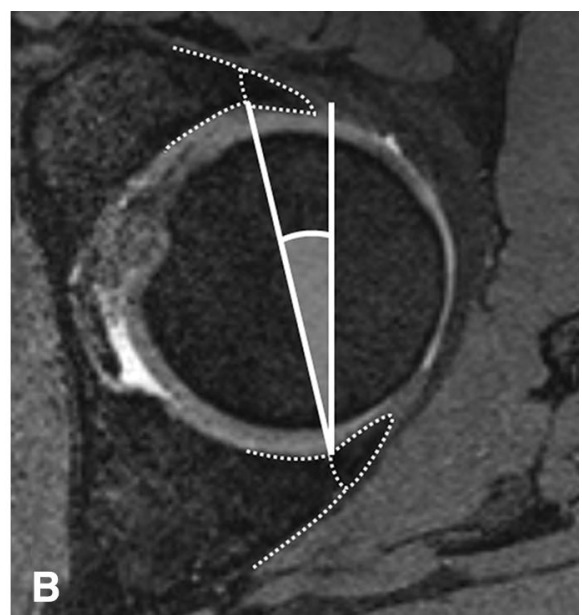

acetabular rim and a sagittal reference line. The reference points for the line connecting both margins of the acetabular rim were defined at the base of the labrum to account for the entire extent of the acetabular socket including non-/partially ossified portions in skeletally (A) immature subjects and (B) mature subjects.

Accordingly, interclass correlation coefficient of the three outcome parameters (acetabular version, acetabular depth/ width ratio, and femoral head coverage) was tested revealing good to excellent intra- and interobserver reliability for all parameters ranging between a mean of 0.85 (range, 0.82$0.87)$ and 0.99 (0.98-1.0 [Table 2]).

We performed a priori power analysis for the primary research question regarding acetabular version related to the status of the TCC with a two-tailed level of significance of $5 \%, \beta$-error of $20 \%$, known acetabular version with of $14^{\circ} \pm 4^{\circ}[9]$ before closure of the TCC, and an estimated acetabular version of $19^{\circ} \pm 4^{\circ}[9]$ after closure of the TCC resulting in a minimum sample size of eight hips. We evaluated all continuous parameters for normal distribution using the Kolmogorov-Smirnov test. Differences among the three groups were assessed with analysis of variance for normally distributed data and the Kruskal-Wallis test for nonnormally distributed data. If differences were found, pairwise comparison was performed with Bonferroni adjustment using the paired t-test for comparisons between measurements obtained from the initial and followup MRI examinations and the independent t-test for measurements among the groups. Accordingly, the Wilcoxon rank-sum test and the Mann-Whitney U-test were applied for pairwise comparison between dependent and independent data, respectively.

\section{Results}

Subjects in the "open-closed" group had decreased acetabular version at the time of the baseline MRI 

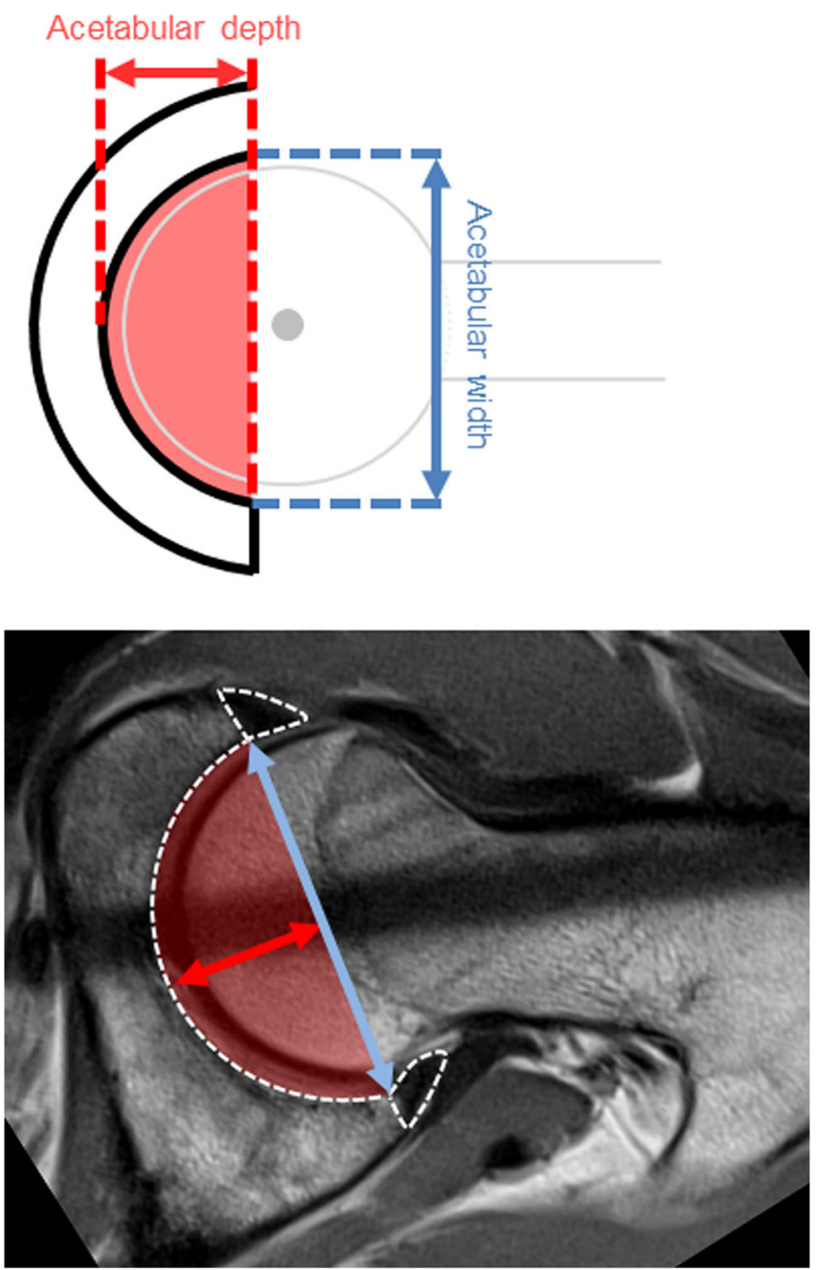

Fig. 5 Acetabular depth/width ratio was assessed three-dimensionally on six radial sequences (Fig. 2). Acetabular depth was measured as the distance between a line connecting the margins of the acetabular rim and a parallel line tangential to the acetabular socket. Acetabular width was the distance of the line connecting the margins of the acetabular rim. Acetabular depth was expressed as the percentage to acetabular width.

examination, which increased as the TCC progressed to complete fusion at the followup MRI examination. Statistical significance was reached at the $5-\mathrm{mm}$ position $\left(-6^{\circ} \pm 4.6^{\circ}\left[95 \%\right.\right.$ confidence interval $\{\mathrm{CI}\},-7.6^{\circ}$ to $\left.-3.6^{\circ}\right]$ versus $-1^{\circ} \pm 5.0^{\circ}\left[95 \% \mathrm{CI},-3.3^{\circ}\right.$ to $\left.0.7^{\circ}\right]$; $\mathrm{p}<0.001)$; the $10-\mathrm{mm}$ position $\left(0^{\circ} \pm 4.0^{\circ}\left[95 \% \mathrm{CI},-1.6^{\circ}\right.\right.$ to $2.1^{\circ}$ ] versus $7^{\circ} \pm 4.6^{\circ}\left[95 \% \mathrm{CI}, 4.4^{\circ}-8.7^{\circ}\right.$ ]; $\mathrm{p}<0.001$ ); and the $15-\mathrm{mm}$ position $\left(8^{\circ} \pm 5.0^{\circ}\left[95 \% \mathrm{CI}, 6.1^{\circ}-10.2^{\circ}\right]\right.$ versus $15^{\circ} \pm 4.6^{\circ}\left[95 \% \mathrm{CI}, 13.3^{\circ}-17.4^{\circ}\right] ; \mathrm{p}<0.001$ ) below the acetabular dome. No difference was observed at the 20 -mm position $\left(14^{\circ} \pm 3.8^{\circ}\left[95 \% \mathrm{CI}, 13^{\circ}-16^{\circ}\right]\right.$ versus $\left.17^{\circ} \pm 3.7^{\circ}\left[95 \% \mathrm{CI}, 16^{\circ}-19^{\circ}\right] ; \mathrm{p}=0.073\right)$ and at the level of the center of the femoral head $\left(15^{\circ} \pm 3.4^{\circ}[95 \% \mathrm{CI}\right.$, $\left.14^{\circ}-17^{\circ}\right]$ versus $18^{\circ} \pm 3.2^{\circ} \quad\left[95 \% \quad \mathrm{CI}, 1^{\circ}-19^{\circ}\right]$; $\mathrm{p}=0.096 ;$ Supplemental Table 1 [Supplemental materials
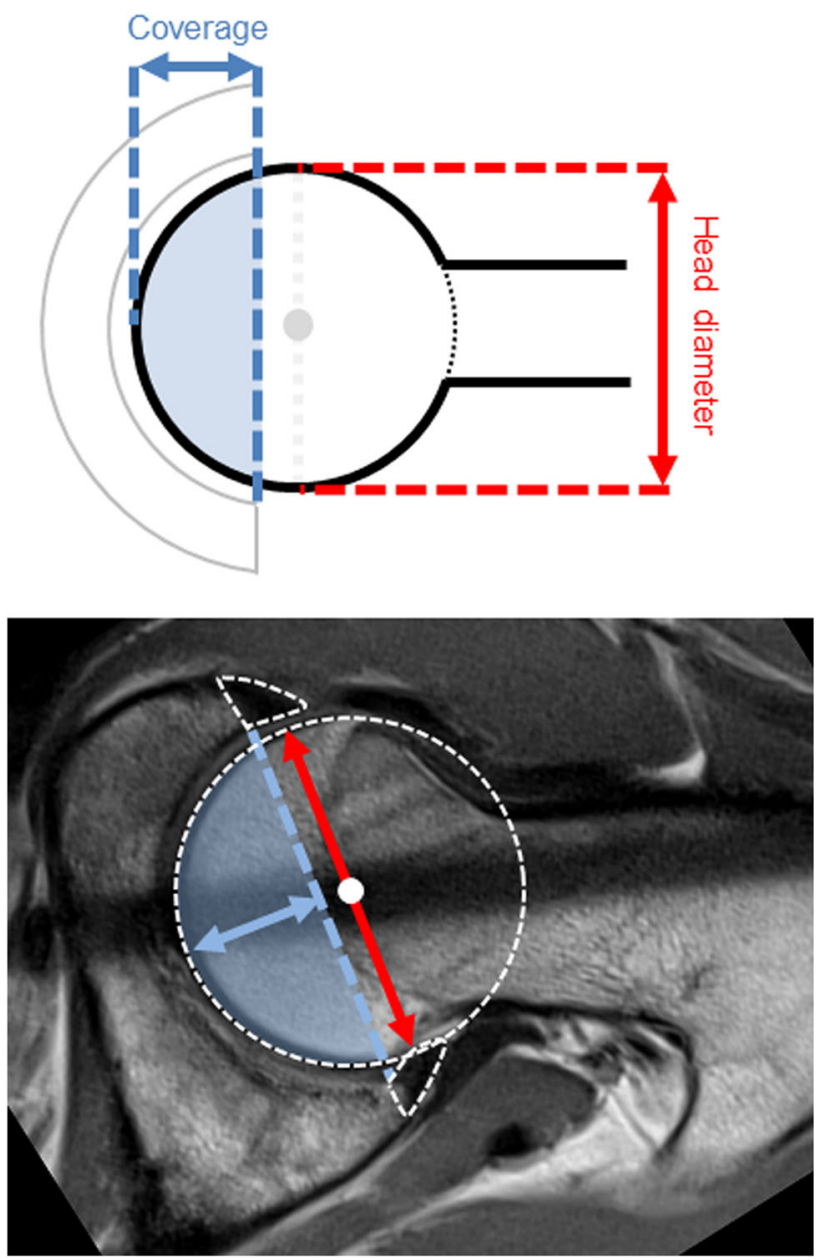

Fig. 6 Three-dimensional femoral head coverage was assessed threedimensionally on six radial sequences (Fig. 2). Femoral head coverage was measured as the distance between a line connecting both margins of the acetabular wall and a parallel line tangential to the femoral head. This distance was expressed as the percentage of the femoral head diameter.

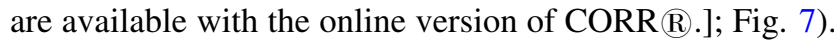
There were no differences in terms of acetabular version between the initial and followup MRI examinations within the "open-open" group (difference measured at $10 \mathrm{~mm}$ below the acetabular dome: $-3^{\circ} \pm 5.5^{\circ}\left[95 \% \mathrm{CI},-4^{\circ}\right.$ to $\left.-1^{\circ}\right]$ at initial MRI versus $-2^{\circ} \pm 5.5^{\circ}\left[95 \% \mathrm{CI},-2^{\circ}\right.$ to $\left.2^{\circ}\right]$ at followup MRI; $\mathrm{p}=0.431$ ) and the "closed-closed" group (difference measured at $10 \mathrm{~mm}$ below acetabular dome: $9^{\circ} \pm 5.9^{\circ}\left[95 \% \mathrm{CI}, 7^{\circ}-11^{\circ}\right]$ at initial MRI versus $9^{\circ} \pm 6.5^{\circ}\left[95 \% \mathrm{CI}, 7^{\circ}-11^{\circ}\right]$ at followup MRI; $p=0.874$ ) at any of the assessed positions (Supplemental Table 1; Fig. 7). Comparison between the groups revealed the lowest values of acetabular version in the "open-open" group (difference measured at $5 \mathrm{~mm}$ below the acetabular dome: $-11^{\circ} \pm 3.1^{\circ}\left[95 \% \mathrm{CI},-12^{\circ}\right.$ to $\left.-10^{\circ}\right]$ at initial MRI and $-9^{\circ} \pm 3.8^{\circ}\left[95 \% \mathrm{CI},-10^{\circ}\right.$ to $-8^{\circ}$ ] at followup MRI; $\mathrm{p}=0.352$ ) followed by increased anteversion in the 
Table 2. Interrater correlation coefficient (ICC) for the three outcome parameters

\begin{tabular}{lllr}
\hline & Intraobserver 1 & Intraoberserver 2 & $\begin{array}{l}\text { Interobserver } \\
\text { ICC }\end{array}$ \\
\hline Acetabular version & ICC & $0.98(0.97-0.99)$ & $0.97(0.95-0.99)$ \\
Acetabular depth/width ratio & $0.99(0.98-1.0)$ & $0.89(0.87-0.91)$ & $0.90(0.88-0.92)$ \\
Femoral head coverage & $0.93(0.91-0.95)$ & $0.84(0.82-0.86)$ & $0.85(0.82-0.87)$ \\
\hline
\end{tabular}

Continuous values are expressed as mean \pm SD with range in parentheses.

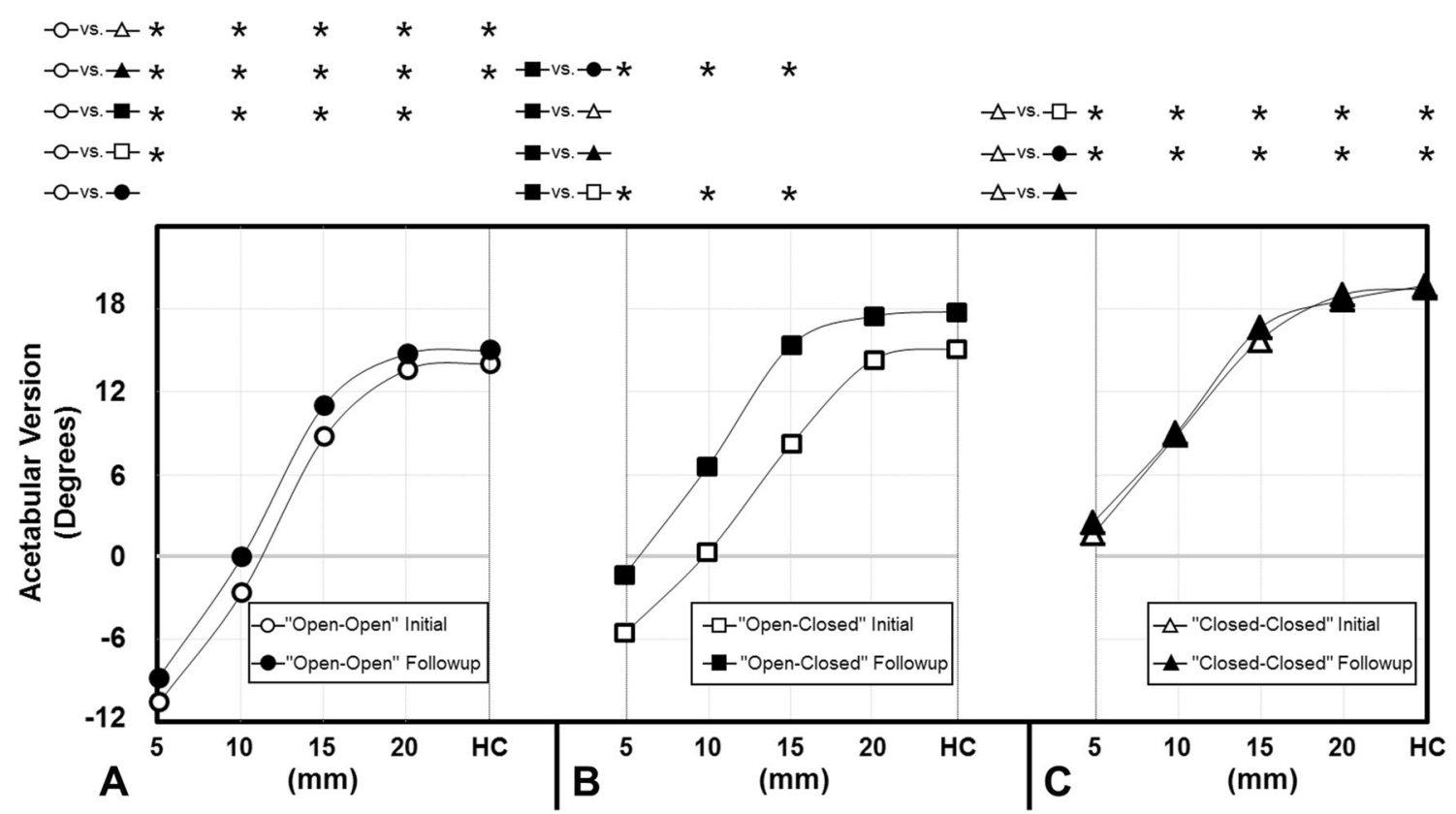

Fig. 7A-C The results of acetabular version for all groups are shown; ${ }^{*} \mathrm{p}<0.05$. All groups revealed increasing acetabular version with increasing distance from the acetabular dome. (A) The lowest values of acetabular version were found for the "open-open" group. There were no differences of acetabular version between the initial and followup MRI examinations. (B) Acetabular version in the "open-closed" group differed between the initial and followup MRI examinations at the 5-mm, 10-mm, and $15-\mathrm{mm}$ positions. Acetabular

"open-closed" group (difference measured at $5 \mathrm{~mm}$ below acetabular dome: $-6^{\circ} \pm 4.7^{\circ}\left[95 \% \mathrm{CI},-8^{\circ}\right.$ to $\left.-4^{\circ}\right]$ at initial MRI and $-1^{\circ} \pm 5.0^{\circ}\left[95 \% \mathrm{CI},-3^{\circ}\right.$ to $\left.1^{\circ}\right]$ at followup MRI; $\mathrm{p}<0.001$ ) and the highest values in the "closed-closed" group (difference measured at $5 \mathrm{~mm}$ below the acetabular dome: $2^{\circ} \pm 5.9^{\circ}\left[95 \% \mathrm{CI}, 0-3^{\circ}\right]$ at initial MRI and $3^{\circ} \pm 5.7^{\circ}\left[95 \% \mathrm{CI}, 1-4^{\circ}\right]$ at followup MRI; $p=0.247$; Supplemental Table 1; Fig. 7). Independently of the status of the TCC, increasing measurement distance from the acetabular dome resulted in increased acetabular version in all groups (Supplemental Table 1; Fig. 7).

There were no differences in the acetabular depth/width ratio among the three groups at any of the measured version in the "open-closed" group at the initial MRI examination did not show any differences compared with the "open-open" group at both MRI examinations. In contrast, acetabular version in the "openclosed" group at the followup examination did not reveal any differences to the "closed-closed" group at both examinations. (C) The "closed-closed" group had the highest values of acetabular version. There were no differences between the initial and followup MRI examinations.

positions (Supplemental Table 2 [Supplemental materials are available with the online version of CORR $\AA$.]; Fig. 8). Both absolute acetabular depth and width increased between the initial and 1-year followup MRI within each group (Supplemental Table 2) resulting in no differences in the depth/width ratio. In general, the acetabular depth/width ratio was lowest in the anterosuperior and corresponding posteroinferior quadrants $(1$ o'clock and 7 o'clock positions) ranging between a mean of $0.45 \pm 0.03(95 \% \mathrm{CI}$, 0.44-0.46) in group "open-closed" at the 1-year followup examination and $0.46 \pm 0.03 \quad(95 \%$ CI, $0.45-0.47$; $\mathrm{p}=0.817$ ) in the "open-open" group at the 1-year followup examination (Fig. 8). At the 3/9 o'clock, 4/10 o'clock, and 5/11 o'clock positions, the acetabular depth/ 


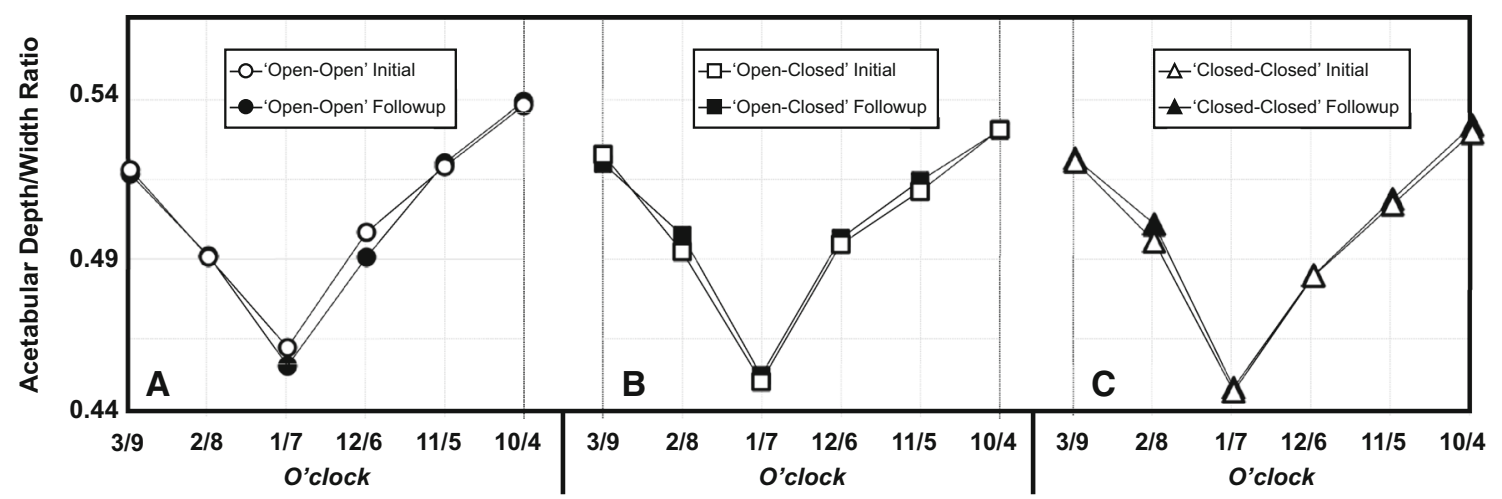

Fig. 8A-C The results of acetabular depth/width ratio are shown for (A) the "open-open" group; (B) the "open-closed" group; and (C) the "closed-closed" group. There were no differences of acetabular

depth/width ratio between the groups. No changes between the initial and followup MRIs were revealed within any of the three groups.

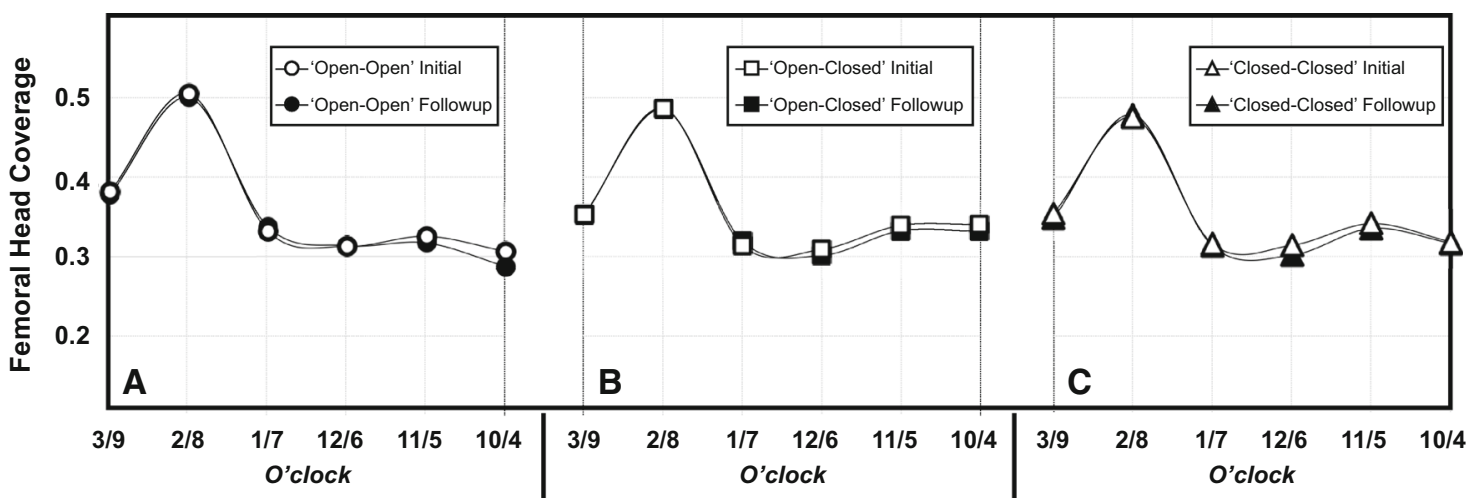

Fig. 9A-C Results of femoral head coverage are shown for (A) the "open-open" group; (B) the "open-closed" group; and (C) the "closed-closed" group. There were no differences of acetabular

width ratio exceeded the value of 0.5 in all groups representing more than a complete hemisphere. The highest depth/width ratio was found in the "open-open" group at the initial MRI examination with a mean of $0.54 \pm 0.04$ (95\% CI, 0.53-0.55; Fig. 8).

Femoral head coverage did not differ among the groups (Supplemental Table 3 [Supplemental materials are available with the online version of CORRß.]; Fig. 9). Furthermore, no differences of femoral head coverage were detected between the initial and 1-year followup MRI examination within each group (Fig. 9). The largest proportion of femoral head coverage was found in the anterosuperior and corresponding posteroinferior quadrants ( 2 o'clock and 8 o'clock positions) in all three groups ranging between a mean of $0.47 \pm 0.04$ (95\% CI, 0.460.48 ) in the "closed-closed" group at the 1-year followup examination and $0.49 \pm 0.06$ (95\% CI, 0.48-0.51; $\mathrm{p}=0.524)$ in the "open-open" group at the 1-year followup examination. femoral head coverage between the groups. No changes between the initial and followup MRIs were revealed within any of the three groups.

\section{Discussion}

The etiology of primary FAI is considered developmental, yet the underlying pathogenic mechanisms remain poorly understood. Although vigorous athletic activity in adolescence has been found to be a risk factor for the development of a cam deformity of the proximal femur $[7,10,11,16,22,23]$, research identifying etiologic factors associated with acetabular retroversion or a deep acetabulum leading to pincer-type FAI is limited [9, 15]. Precise knowledge of the physiologic patterns of the developing acetabulum during skeletal maturation may help to explain developmental disorders of the hip. Serial MRI allows noninvasive 3-D assessment while simultaneously depicting the true dimensions of the maturing acetabulum including the cartilaginous portions in skeletally immature subjects. The aims of this study were to assess morphologic parameters that might contribute to pincer-type FAI based on two serial MRI examinations obtained with a minimum 
interval of 1 year in a population of asymptomatic volunteers with no known hip pathology. The specific study question was: Are there any differences related to skeletally maturity with regard to (1) acetabular version; (2) acetabular depth/width ratio; and (3) femoral head coverage in the same children as assessed by MRIs obtained 1 year apart?

This study has limitations. First, the assessment of acetabular version was not standardized for pelvic tilt and rotation. Because all MRIs were obtained for each hip separately, it was not possible to correct for pelvic orientation. To counteract malpositioning and subsequent pelvic malorientation during image acquisition, all study participants were positioned supine, feet first, and the legs in holding devices according to a standardized protocol. It has previously been shown that there are no individual side differences in terms of acetabular version [3, 27, 31]. Given the fact that all but one participant received bilateral MRIs of the hip at both examinations (Table 1), malrotation of the pelvis resulting in increased version on one side would have been compensated by decreased version on the other side. We attempted to evaluate the effect of malrotation by extrapolating side-related, intraindividual variations of acetabular version in all 64 study participants (128 MRIs). We found minor intraindividual side differences with a mean of $2.9^{\circ} \pm 2.1^{\circ}$ (range, $0-9^{\circ} ; 95 \% \mathrm{CI}, 2.7-3.1$ ) at the initial MRI and of $2.7^{\circ} \pm 2.1^{\circ}$ (range, $0-9^{\circ}$; 95\% CI, 2.5$29^{\circ}$ ) at followup. However, we cannot entirely rule out malorientation of the pelvis, and it remains a limitation of the study.

In addition, we only assessed the development of the acetabulum itself, thereby neglecting the interaction of the femoral head on the development of the acetabulum. It has previously been shown that the sphericity of the femoral head is directly related to the concavity of the acetabulum $[2,8,17]$. However, none of the participants had a history of previous hip disorder. Additionally, no gross asphericities of the femoral head were found in this population in a previous study [12]. Another limitation is the relatively homogenous population of study participants. All volunteers were from the same school and all were white. Thus, data obtained from this study might not be representative of the general population. Furthermore, the number of hips allocated to the "open-closed" group was relatively small. The resulting lack of power limited the ability to determine sex differences within the groups. Also, although all study participants denied hip symptoms and none had a history of a previous hip disorder, our findings were neither correlated with a clinical history about lifestyle factors or physical activity level nor with a dedicated clinical examination. Both may have a confounding influence on the development of the acetabulum. Finally, the followup period of 1 year is relatively short. A longer followup period might have revealed more pronounced intraindividual differences within the groups. Additionally, a larger number of participants with an initially open TCC would have progressed to complete fusion, thereby increasing the power in the "open-closed" group.

We found distinct differences of acetabular version related to skeletal maturity. Version was decreased in hips with open TCC and increased with closed TCC. Acetabular version changed within the "open-closed" group from lower version at the initial examination (TCC open) to more pronounced anteversion at the 1-year followup examination (TCC closed) at the 5-mm, 10- $\mathrm{mm}$, and 15 $\mathrm{mm}$ positions. Our findings indicate a close relationship between acetabular version and maturation of the acetabulum that changes within a narrow timeframe of 1 year as the TCC progresses to complete fusion. Previous studies have investigated acetabular orientation related to skeletal maturity (Table 3). Hingsammer et al. [9] found decreased anteversion in skeletally immature compared with skeletally mature subjects. Another study reported no substantial differences of acetabular version in children younger than 11 years, but a progressive increase of anteversion was noted in patients older than 12 years of age [15]. In contrast, Weiner et al. [29] reported no age-dependent differences of acetabular version in a population ranging from 6 months to 17 years and similar results were found in another study that compared normal and dysplastic hips during skeletal maturation [13]. However, it should be noted that all these studies were based on CT measurements, which cannot measure the true dimensions of the acetabulum because the cartilaginous portions of the acetabular wall in skeletally immature subjects are underrepresented. A recent study reported that posterior wall ossification lagged behind anterior wall ossification [4]. This finding has a direct influence on CT-derived measurements performed at the acetabular margins in skeletally immature subjects. Because we used MRI-derived data, the complete extent of the acetabular wall was depicted (Fig. 4) and underrepresentation of nonossified portions of the acetabular rim can be precluded.

We found no differences in terms of the acetabular depth/width ratio depending on the status of the TCC. Additionally, there were no changes between the baseline and followup MRI examinations. The 3-D assessment revealed a characteristic pattern of the depth/width ratio with the shallowest acetabular socket at the $2 / 8$ o'clock, $1 / 7$ o'clock, and 12/6 o'clock positions. A socket exceeding the dimensions of a complete hemisphere was found at the 3/9 o'clock, 4/10 o'clock, and 5/11 o'clock positions. The growth of acetabular depth and width relies on the interstitial growth of the TCC. Rális and McKibbin [19] investigated the acetabular depth/width ratio in 44 specimens from 15 fetuses and 29 children ranging from 


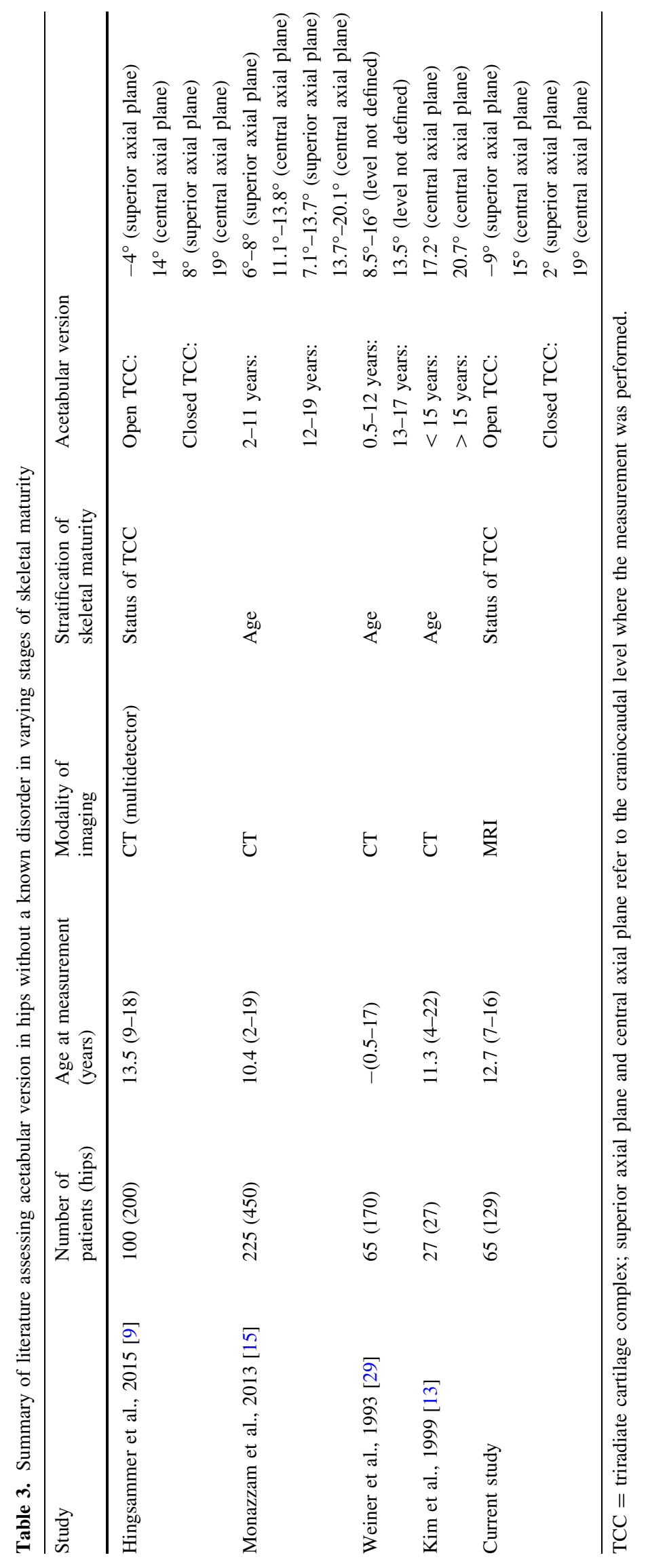


12 weeks of gestation to 11 years of age. Throughout fetal life, acetabular depth/width ratio continuously decreased toward birth at which the shallowest shape was present. This trend reversed after birth with an increasingly deepened socket until the age of 11 years [19]. In contrast to our study, Monazzam et al. [15] assessed the acetabular depth/ width ratio based on coronal CT slices (according to the 12/ 6 o'clock position) in skeletally mature and immature patients and reported an increasing depth/width ratio with increasing age.

We found no differences in femoral head coverage among the groups. There were also no differences of coverage between the baseline and 1-year followup examinations. Changes of femoral head coverage during skeletal maturation have been extensively studied in the setting of developmental dysplasia of the hip [14, 18, 19]. However, there is little research addressing growth patterns of the developing acetabulum regarding overcoverage. Previous measurements of acetabular coverage based on CT data in skeletally mature and immature subjects (age range, 2-19 years) revealed a higher prevalence of overcoverage (defined as a lateral center-edge angle $>40^{\circ}$ [30] and a Tönnis angle $<0^{\circ}$ [28]) with increasing age [15]. A study investigating acetabular coverage using acetabular sector angles [1,5] in skeletally mature and immature subjects using CT found increased coverage at the superior, posterosuperior, and posterior positions in subjects with closed TCC compared with open TCC in both males and females [9], whereas no differences were detected at the anterior and anterosuperior positions. It was concluded that posterior wall deficiency in skeletally immature patients accounts for acetabular retroversion. This contrasts with our findings, which suggest that acetabular retroversion is not only the result of posterior wall deficiency, but represent true malorientation of the acetabulum. This is supported by Steppacher et al. [25], who found no differences in shape and size of the lunate fossa between retroverted and normal hips, which would be expected if retroversion is the result of posterior wall deficiency.

In summary, this study used MRI to longitudinally assess pincer-type lesions in the developing acetabulum and revealed a typical pattern related to skeletal maturity; whereas the acetabular depth/width ratio and femoral head coverage appear to be constant immediately before and after closure of the TCC, it was shown that acetabular version increased with increasing maturity of the acetabulum. There seems to be a narrow timeframe near closure of the TCC within which acetabular orientation progresses to more pronounced anteversion. Underrepresentation of the nonossified portions of the acetabular rim in skeletally immature subjects can be precluded as the entire extent of the acetabular wall was depicted using MRI. The fact that acetabular version increased while acetabular depth/width ratio and femoral head coverage remained unchanged is perhaps more suggestive of a rotational mechanism during this late phase of maturation than relative growth differences between the anterior and posterior wall. Conditions leading to premature closure of the TCC might inhibit the progression to more pronounced acetabular anteversion ultimately contributing to the development of pincer-type FAI. Because there were no differences in acetabular depth/width ratio and femoral head coverage related to skeletal maturity, our data do not shed light on potential mechanisms for the development of general overcoverage as the underlying cause for pincer-type FAI. Further studies with serial MRI examinations and longer followup periods are required to support these findings.

\section{References}

1. Anda S, Svenningsen S, Dale LG, Benum P. The acetabular sector angle of the adult hip determined by computed tomography. Acta Radiol Diagn (Stockh). 1986;27:443-447.

2. Bucholz RW, Ezaki M, Ogden JA. Injury to the acetabular triradiate physeal cartilage. J Bone Joint Surg Am. 1982;64:600-609.

3. Buller LT, Rosneck J, Monaco FM, Butler R, Smith T, Barsoum WK. Relationship between proximal femoral and acetabular alignment in normal hip joints using 3-dimensional computed tomography. Am J Sports Med. 2012;40:367-375.

4. Fabricant PD, Hirsch BP, Holmes I, Kelly BT, Lorich DG, Helfet DL, Bogner EA, Green DW. A radiographic study of the ossification of the posterior wall of the acetabulum: implications for the diagnosis of pediatric and adolescent hip disorders. J Bone Joint Surg Am. 2013;95:230-236.

5. Fujii M, Nakashima Y, Yamamoto T, Mawatari T, Motomura G, Matsushita A, Matsuda S, Jingushi S, Iwamoto Y. Acetabular retroversion in developmental dysplasia of the hip. J Bone Joint Surg Am. 2010;92:895-903.

6. Ganz R, Parvizi J, Beck M, Leunig M, Notzli H, Siebenrock KA. Femoroacetabular impingement: a cause for osteoarthritis of the hip. Clin Orthop Relat Res. 2003;417:112-120.

7. Gerhardt MB, Romero AA, Silvers HJ, Harris DJ, Watanabe D, Mandelbaum BR. The prevalence of radiographic hip abnormalities in elite soccer players. Am J Sports Med. 2012;40:584-588.

8. Harrison TJ. The influence of the femoral head on pelvic growth and acetabular form in the rat. J Anat. 1961;95:12-24.

9. Hingsammer AM, Bixby S, Zurakowski D, Yen YM, Kim YJ. How do acetabular version and femoral head coverage change with skeletal maturity? Clin Orthop Relat Res. 2015;473:1224-1233.

10. Johnson AC, Shaman MA, Ryan TG. Femoroacetabular impingement in former high-level youth soccer players. Am J Sports Med. 2012;40:1342-1346.

11. Kapron AL, Peters CL, Aoki SK, Beckmann JT, Erickson JA, Anderson MB, Pelt CE. The prevalence of radiographic findings of structural hip deformities in female collegiate athletes. Am J Sports Med. 2015;43:1324-1330.

12. Kienle KP, Keck J, Werlen S, Kim YJ, Siebenrock KA, Mamisch TC. Femoral morphology and epiphyseal growth plate changes of the hip during maturation: MR assessments in a 1-year follow-up on a cross-sectional asymptomatic cohort in the age range of 9-17 years. Skeletal Radiol. 2012;41:1381-1390.

13. Kim SS, Frick SL, Wenger DR. Anteversion of the acetabulum in developmental dysplasia of the hip: analysis with computed tomography. J Pediatr Orthop. 1999;19:438-442. 
14. Lee MC, Eberson CP. Growth and development of the child's hip. Orthop Clin North Am. 2006;37:119-132, v.

15. Monazzam S, Bomar JD, Dwek JR, Hosalkar HS, Pennock AT. Development and prevalence of femoroacetabular impingementassociated morphology in a paediatric and adolescent population: a CT study of 225 patients. Bone Joint J. 2013;95:598-604.

16. Nepple JJ, Brophy RH, Matava MJ, Wright RW, Clohisy JC. Radiographic findings of femoroacetabular impingement in National Football League combine athletes undergoing radiographs for previous hip or groin pain. Arthroscopy. 2012;28: 1396-1403.

17. Ponseti IV. Growth and development of the acetabulum in the normal child. Anatomical, histological, and roentgenographic studies. J Bone Joint Surg Am. 1978;60:575-585.

18. Portinaro NM, Porteous A, Parafioriti A, Panou A, Benson MK. Growth of the acetabular lateral cartilage in relation to congenital and developmental dysplasia of the hip. An histological study. Hip Int. 2011;21:9-13.

19. Rális Z, McKibbin B. Changes in shape of the human hip joint during its development and their relation to its stability. $J$ Bone Joint Surg Br. 1973;55:780-785.

20. Reynolds D, Lucas J, Klaue K. Retroversion of the acetabulum. A cause of hip pain. J Bone Joint Surg Br. 1999;81:281-288.

21. Rubel IF, Kloen P, Potter HG, Helfet DL. MRI assessment of the posterior acetabular wall fracture in traumatic dislocation of the hip in children. Pediatr Radiol. 2002;32:435-439.

22. Siebenrock KA, Behning A, Mamisch TC, Schwab JM. Growth plate alteration precedes cam-type deformity in elite basketball players. Clin Orthop Relat Res. 2013;471:1084-1091.
23. Siebenrock KA, Ferner F, Noble PC, Santore RF, Werlen S, Mamisch TC. The cam-type deformity of the proximal femur arises in childhood in response to vigorous sporting activity. Clin Orthop Relat Res. 2011;469:3229-3240.

24. Stasikelis PJ, Sullivan CM, Phillips WA, Polard JA. Slipped capital femoral epiphysis. Prediction of contralateral involvement. J Bone Joint Surg Am. 1996;78:1149-1155.

25. Steppacher SD, Lerch TD, Gharanizadeh K, Liechti EF, Werlen SF, Puls M, Tannast M, Siebenrock KA. Size and shape of the lunate surface in different types of pincer impingement: theoretical implications for surgical therapy. Osteoarthritis Cartilage. 2014;22:951-958.

26. Strayer LM Jr. Embryology of the human hip joint. Clin Orthop Relat Res. 1971;74:221-240.

27. Tallroth K, Lepisto J. Computed tomography measurement of acetabular dimensions: normal values for correction of dysplasia. Acta Orthop. 2006;77:598-602.

28. Tönnis D, Heinecke A. Acetabular and femoral anteversion: relationship with osteoarthritis of the hip. J Bone Joint Surg Am. 1999;81:1747-1770.

29. Weiner LS, Kelley MA, Ulin RI, Wallach D. Development of the acetabulum and hip: computed tomography analysis of the axial plane. J Pediatr Orthop. 1993;13:421-425.

30. Wiberg G. The anatomy and roentgenographic appearance of a normal hip joint. Acta Chir Scand. 1939;83:7-38.

31. Zeng Y, Wang Y, Zhu Z, Tang T, Dai K, Qiu S. Differences in acetabular morphology related to side and sex in a Chinese population. J Anat. 2012;220:256-262. 